\title{
Efficient Minimum-Phase Prefilter Computation Using Fast QL-Factorization
}

\author{
Hansen, Morten; Christensen, Lars P.B.
}

Published in:

IEEE International Conference on Acoustics, Speech and Signal Processing, 2009. ICASSP 2009.

Link to article, DOI:

10.1109/ICASSP.2009.4960179

Publication date:

2009

Document Version

Publisher's PDF, also known as Version of record

Link back to DTU Orbit

Citation (APA):

Hansen, M., \& Christensen, L. P. B. (2009). Efficient Minimum-Phase Prefilter Computation Using Fast QLFactorization. In IEEE International Conference on Acoustics, Speech and Signal Processing, 2009. ICASSP 2009. IEEE. https://doi.org/10.1109/ICASSP.2009.4960179

\section{General rights}

Copyright and moral rights for the publications made accessible in the public portal are retained by the authors and/or other copyright owners and it is a condition of accessing publications that users recognise and abide by the legal requirements associated with these rights.

- Users may download and print one copy of any publication from the public portal for the purpose of private study or research.

- You may not further distribute the material or use it for any profit-making activity or commercial gain

- You may freely distribute the URL identifying the publication in the public portal 


\section{EFFICIENT MINIMUM-PHASE PREFILTER COMPUTATION USING FAST QL-FACTORIZATION}

\section{Morten Hansen}

Technical University of Denmark, Informatics and Mathematical Modelling, Build. 321, DK-2800 Lyngby, Denmark, E-mail:mha@imm.dtu.dk.

\section{Lars P. B. Christensen}

\author{
Nokia Denmark \\ Frederikskaj 5, \\ DK-1790 Copenhagen V, Denmark, \\ E-mail: lars.christensen@nokia.com.
}

\begin{abstract}
This paper presents a novel approach for computing both the minimum-phase filter and the associated all-pass filter in a computationally efficient way using the fast QL-factorization. A desirable property of this approach is that the complexity is independent on the size of the matrix which is QL-factorized, and thereby the complexity scales with the required precision of the filters and the filter length.
\end{abstract}

Index Terms - Communications, prefiltering, minimumphase systems, fast QL-factorization.

\section{INTRODUCTION}

The minimum-phase filter has during the years gained much attention and since it is applied in various fields, it is often treated in general signal processing books, [1]. One place where the minimum-phase filter plays an important role is in communication systems when higher-order modulation schemes over multipath channels are used. In such systems, optimal sequence detection can be obtained using MaximumLikelihood Sequence Estimation (MLSE), but MLSE will however require an unacceptable high complexity for channels with large delay spread (i.e. long impulse responses). Therefore, other suboptimal techniques such as delayed decision feedback, or reduced-state sequence estimation, will often be used in such systems [2]. To obtain reliable detection using these techniques, both the minimum-phase and the associated all-pass filter are used.

In this paper we describe a new approach for efficiently computing the minimum-phase filter and the all-pass filter by performing a fast QL-factorization of the channel matrix. The paper is organized as follows; In Section 2 we present the signal model, and Section 3 describes the connection between the minimum-phase filter and the QL-factorization. In Section 4 we illustrate how the fast QL-factoization can be utilized for time-invariant channels, while the simulation results are found in 5. Finally Section 6 contains some concluding remarks.

\section{SYSTEM MODEL}

Consider a time-invariant Single-Input Single-Output (SISO) system $^{1}$, which can be described by the Finite Impulse Response (FIR) filter, $\mathcal{H}$, having the length $L$. The output signal $y_{k} \in \mathbb{C}$ at time index $k$ can be expressed as

$$
y_{k}=\sum_{l=0}^{L-1} h_{l} x_{k-l},
$$

where $x_{k} \in \mathbb{C}$ is the input signal at time index $k=\{1,2, \ldots, N+L-1\}, N$ is the length of the input sequence, and $h_{l} \in \mathbb{C}$ denotes the l'th tap in the impulse response. Using matrix notation, the system model in (1) can be formulated as

$$
\mathbf{y}=\mathbf{H x}
$$

where $\mathbf{y}=\left[\begin{array}{llll}y_{1}, & y_{2}, \ldots, & y_{N+L-1}\end{array}\right]^{T}$ and $\mathbf{x}=\left[\begin{array}{llll}x_{1}, & x_{2}, & \ldots, & x_{N}\end{array}\right]^{T}$. To ease the notation let $M \triangleq(N+L-1)$, leading to $\mathbf{y} \in \mathbb{C}^{M}$. Due to the timeinvariant property of the filter, $\mathbf{H} \in \mathbb{C}^{M \times N}$ will be a banded Toeplitz convolution matrix having the form

$$
\mathbf{H} \triangleq\left[\begin{array}{cccc}
h_{0} & 0 & \cdots & 0 \\
\vdots & h_{0} & \ddots & \vdots \\
h_{L-1} & \ddots & \ddots & 0 \\
0 & \ddots & \ddots & h_{0} \\
\vdots & \ddots & \ddots & \vdots \\
0 & \cdots & 0 & h_{L-1}
\end{array}\right] .
$$

In the analysis of the filter characteristic, it is often useful to z-transform the channel impulse response [1], which leads to

$$
H(z)=\sum_{l=0}^{L-1} h_{l} z^{-l} .
$$

\footnotetext{
${ }^{1}$ Results presented in this paper may be directly extended to MultipleInput Multiple-Output (MIMO) systems, but this is outside the scope of this paper.
} 
A classical way of obtaining the minimum-phase filter, $H_{m i n}$, is by using the root method of spectral factorization, where we first find roots in the polynomial given in (3), and reflect the roots located outside the unit circle, into the circle, [1], [3]. Based on the roots inside and on the unit circle, a new polynomial can be computed in the z-domain, which represents the minimum-phase filter. There exists however several other spectral factorization methods which among others is described in [4]. In many applications (e.g. in communications) we also need the associated all-pass filter, which is used to prefilter the input signal, $\mathbf{x}$, such that output signal matches the minimum-phase filter. As finding the minimumphase and all-pass filters can be computationally expensive, approximative methods having lower complexity may be of practical interest [2].

\section{CONNECTION BETWEEN THE MINIMUM-PHASE FILTER AND THE QL-FACTORIZATION}

Recently, it has been discovered that the minimum-phase filter and its associated all-pass filter can be obtained by performing a QL-factorization of the channel matrix, H, [5], [6]. When we perform the factorization,

$$
\mathbf{H}=\mathbf{Q} \tilde{\mathbf{L}}=\mathbf{Q}\left[\begin{array}{c}
\mathbf{0}_{(M-N) \times N} \\
\mathbf{L}
\end{array}\right]
$$

we require that the $N \times N$ lower triangular matrix, $\mathbf{L}$, corresponds to the Cholesky factor of $\mathbf{H}^{H} \mathbf{H}$, meaning that $\mathbf{L}$ is positive definite and contains real-valued positive diagonal elements (assuming that $\operatorname{rank}(\mathbf{H})=N$ ). Since we perform a factorization of a banded Toeplitz matrix, each row in $\mathbf{L}$ will be a shifted version of each other as $\{M, N\} \rightarrow \infty$, and each row is precisely given by the spectral factorization, [7]. Likewise, the $M \times M$ unitary matrix $\mathbf{Q}$ will be the matrix equivalent of the all-pass filter and again each column of $\mathbf{Q}$ will be a shifted version of each other. Furthermore, it can be seen that each of these columns will correspond to the all-pass filter associated with the minimum-phase filter. For a detailed description of this, see [5], [6].

In the finite length case, each row of $\mathbf{L}$ (column of $\mathbf{Q}$ ) will not be exactly the same, but as can be seen in [6], the values in each row of $\mathbf{L}$ will converge toward the true minimum-phase filter as a function of the row number ${ }^{2}$, likewise the columns of $\mathbf{Q}$ will converge toward the associated all-pass filter. Thus, the accuracy of the estimated filter coefficients (compared to the true filters) depends on where in $\mathbf{L}$ and $\mathbf{Q}$ we take out the filter coefficients.

\footnotetext{
${ }^{2}$ Using the Householder method, elements of rows in $\mathbf{L}$ converge toward the minimum-phase filter from the bottom and up due to elements in the lower triangular matrix being computed from the bottom and up.
}

\section{FAST QL-FACTORIZATION}

When traditional methods are used to compute the QLfactorization it requires $\mathcal{O}\left(N^{3}\right)$ operations, [8], but for Toeplitz matrices there exist methods with lower computational complexity. Different methods have been proposed for performing the fast QL-factorization ${ }^{3}$ [8], [9], [10], each of which has different numerical properties and slightly different complexity as well. They do however all use the shift-invariance property of Toeplitz matrices to partition it in two ways, and it is this partitioning that leads to the low complexity schemes. In [8], the QL-factorization can be performed using $13 M N+6 N^{2}$ operations for general $M \times N$ Toeplitz matrices, while the method proposed in [10] require $13 M N+6.5 N^{2}$. The methods described in [8], [9], and [10] all deal with real-valued matrices, but the results can be extended to be valid over the complex field, [10]. Furthermore, the methods can be extended to handle block Toeplitz matrices for the general MIMO case as well, [11].

The fast QL-factorization computes a single row of $\mathbf{L}$ (or column of $\mathbf{Q}$ ) at a time, which turns out to be a great advantage when the QL-factorization is used for prefilter computation. This is due to the fact that each row of $\mathbf{L}$ converges toward the true minimum-phase filter, which implies that we can stop the computation of the rows in $\mathbf{L}$ once we have obtained the required precision of the filter coefficients. Likewise, we only need to compute a certain fraction of the columns in $\mathbf{Q}$ to obtain the required precision of the all-pass filter. Thus, by using the fast QL-factorization to compute the filters, the complexity no longer scales with the size of the matrix, $\mathbf{H}$, but depends on the required precision. The number of rows in $\mathbf{L}$ (and thus columns in $\mathbf{Q}$ ) which is used to obtain the estimated minimum-phase and all-pass filters, is referred to as the number of iterations, $n$.

The complexity of the fast QL-factorization can be reduced even further, using the fact that the Toeplitz channel matrix, $\mathbf{H}$, contains at most $L$ non-zero elements in each row. Thus, using the method described in [8], we can compute of each row in $\mathbf{L}$ using $4 L+7$ complex operations and two square root computations. On top of that we also need to take into account the initialization step, which determines the bottom row of $\mathbf{L}^{4}$, requiring $(L-1)^{2}+4 L$ complex operations and two square root computations. Thus, if the required precision of the minimum-phase estimate can be obtained using $n$ iterations, the computational complexity will be

$$
\mathcal{O}_{\text {min }}=n \cdot(4 L+7)+(L-1)^{2}+4 L,
$$

complex operations plus $2 n+2$ square root operations. Each of the last $L_{a p}-L$ columns of $\mathbf{Q}$ require $(L+i)(i+1)$ operations where $i=0, \ldots, L_{a p}-L-1$. The complexity of computing each of the $j$ last columns of $\mathbf{Q}$ is $L_{a p}(j+1)$

\footnotetext{
${ }^{3}$ Methods for QR-factorization may easily be converted to QL.

${ }^{4}$ The QL-factorization starts from the bottom row and works its way up to the top, while the QR-factorization uses a top down approach.
} 


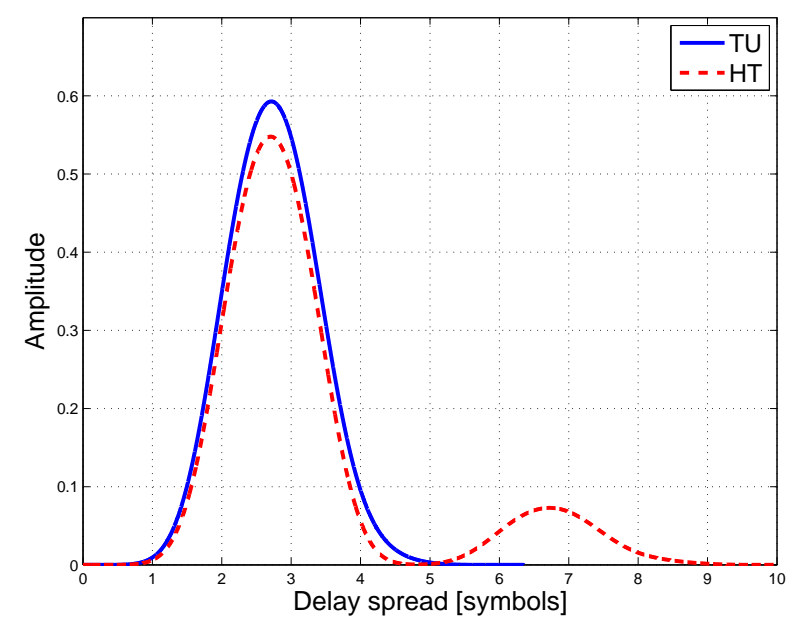

Fig. 1. Channel profiles of Typical Urban and Hilly Terrain (including the transmit pulse shaping).

for $j=L_{a p}-L+1, \ldots, L_{a p}$. If the number of iterations needed is higher than the length of the prefilter, we also need $L_{a p}\left(L_{a p}+1\right)$ complex operations to calculate each of the remaining columns (i.e. the columns from $L_{a p}+1$ to $n$ ). Thus, the overall complexity of computing the prefilter, is

$$
\begin{gathered}
\mathcal{O}_{a p}=\quad \sum_{k=0}^{\min \left\{\left(L_{a p}-1\right) ;(n-1)\right\}} \min \left\{(L+k) ; L_{a p}\right\} \cdot(k+1) \\
+\max \left\{0 ;\left(n-L_{a p}\right)\right\} \cdot L_{a p}\left(L_{a p}+1\right)
\end{gathered}
$$

assuming that $n \geq L_{a p}-L+1$. Note that the last term in (6) vanishes when $n \leq L_{a p}$ and that we will obtain the first $L_{a p}$ filter coefficients after $\left(L_{a p}-L+1\right)$ iterations. Thus, in cases where $L$ is close to $L_{a p}$ we only need a few iterations if we are willing to sacrifice precision in favor of complexity. Thus, for the Hilly Terrain (HT0) profile specified in [12], the minimum-phase filter and the all-pass filters can be obtained using 546 operations (where $L=10$ and using $L_{a p}=14$, $n=5)$.

The approximate low complexity method proposed in [2], which uses Linear Prediction (LP) to obtain an estimate of the all-pass and minimum-phase filters, will approximately require $1 / 2 \cdot(L+1)(L+2)+L_{p}^{2}+2 L_{p}+(L+1)\left(L_{p}+1\right)$ operations (complex multiplications). Here $L_{p}$ denotes the order of the prediction-error filter. When $L_{p}=14$ this method requires 455 operations for the HT0 profile. Thus, for some practical channel profiles the method proposed in this paper will have a complexity which almost is in the same scale as the low complexity LP-method.

\section{SIMULATION RESULTS}

In this section we present simulation results for 3 different types of SISO channels. First we assume that we have complex Gaussian distributed, $\mathcal{C N}(0,1)$, channel coefficients. Secondly, we consider two types of channels defined in the GSM specifications [12], namely the Typical Urban (TU0)

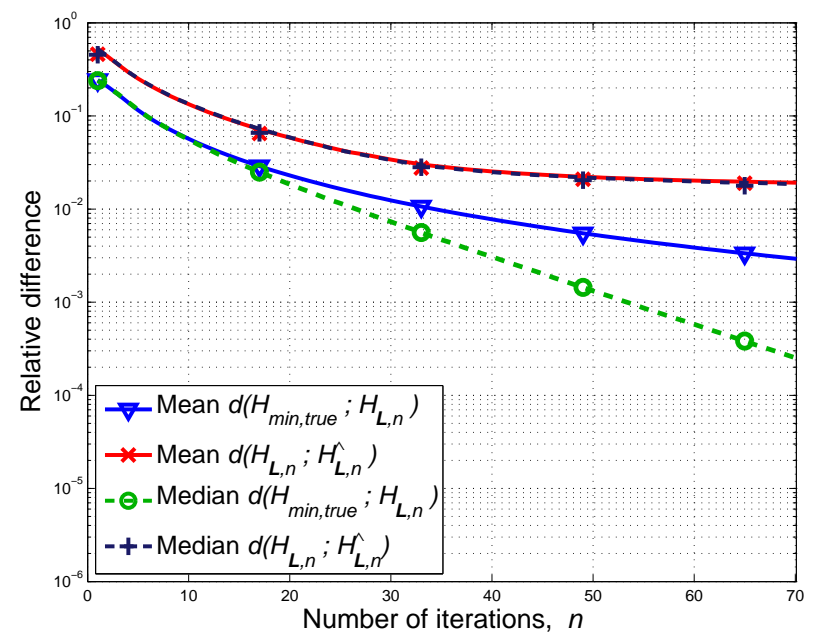

Fig. 2. Gaussian filter coefficients, $L=7$. Mean and median value of the relative deviations, $d\left(\mathcal{H}_{\text {min,true }} ; \mathcal{H}_{L, n}\right)$ and $d\left(\mathcal{H}_{L, n} ; \widehat{\mathcal{H}}_{L, n}\right)$ when $L_{a p}=32$.

and the Hilly Terrain (HT0) profiles, which can also be seen in Fig. 1.

We compute the relative difference between the two filters, $\mathcal{H}_{a}$ and $\mathcal{H}_{b}$, as a function of the iteration number, $n$, as

$$
d\left(\mathcal{H}_{a, n} ; \mathcal{H}_{b, n}\right) \triangleq \frac{\left\|\mathcal{H}_{a, n}-\mathcal{H}_{b, n}\right\|_{2}}{\left\|\mathcal{H}_{a, n}\right\|_{2}}
$$

which is done in order to measure the convergence rate of the filter coefficients. In the simulations $\mathcal{H}_{\text {min,true }}$ denotes the impulse response of the true minimum-phase filter, and $\mathcal{H}_{L, n}$ is the impulse response obtained from $\mathbf{L}$ (at iteration $n$ ). To measure how well the estimated all-pass filter, $\mathcal{H}_{Q, n}$, match the estimated minimum-phase filter $\mathcal{H}_{L, n}$, we filter the original impulse response $\mathcal{H}$ with $\mathcal{H}_{Q, n}^{*}$, which gives us the output $\widehat{\mathcal{H}}_{L, n}$. In all the simulations presented below, we have made 10000 realizations of the examined channel profile, and computed the minimum-phase and the all-pass filter for each realization. The filter length of the all-pass filter is in all simulations $L_{a p}=32$. Based on the result of the 10000 filter realizations, we have computed the mean and median value of the relative errors, $d\left(\mathcal{H}_{\text {min,true }} ; \mathcal{H}_{L, n}\right)$ and $d\left(\mathcal{H}_{L, n} ; \widehat{\mathcal{H}}_{L, n}\right)$. The result for the Gaussian channel coefficients is shown in Fig. 2, where we see that the convergence toward the true minimum-phase filter is exponential. In Fig. 3 the result for the TU0 profile is shown, and here we can see that the average relative deviation between the true minimum-phase filter and estimated solution is approximately $10^{-2}$ after $7-8$ iterations. To obtain the same relative deviation between the estimated minimum-phase filter and the estimated all-pass filter we need approximately 14-15 iterations. We can see from the figure that the median value of the relative error converges faster than the mean value, which indicates that some of the realizations will bias the estimate of the mean value due to "out- 


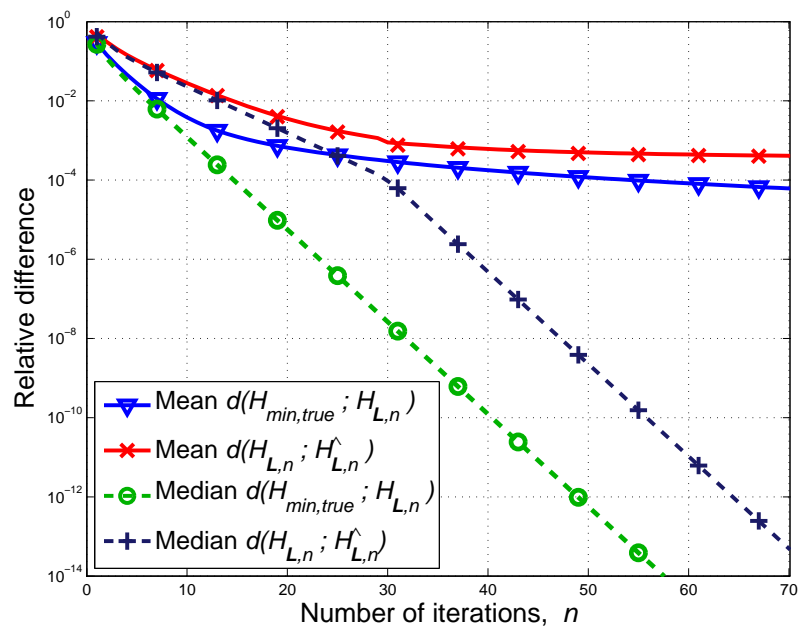

Fig. 3. TU0 profile, $L=6$. Mean and median value of the relative deviations, $d\left(\mathcal{H}_{\text {min }, \text { true }} ; \mathcal{H}_{L, n}\right)$ and $d\left(\mathcal{H}_{L, n} ; \widehat{\mathcal{H}}_{L, n}\right)$ when $L_{a p}=32$.

liers" in the distribution of the relative error. By inspecting the approximated PDF for different iterations, it is observed that a few realizations converge slower than the majority, and they will therefore in some sense bias the estimate. The realizations which converge slowest are the ones which contain roots located close to the unit circle. Fig. 4 show the result for the HT0 profile, and in this case the convergence is slower than the TU0 profile. This is not surprising, since the channel impulse response of HT profile is longer, which makes it more likely that there are roots close to the unit circle. For this profile we need 21 iterations to obtain an average precision of $10^{-2}$ between the true and estimated minimum-phase filter. In Fig. 2 to Fig. 4 we see that the relative difference $d\left(\mathcal{H}_{L, n} ; \widehat{\mathcal{H}}_{L, n}\right)$ tends to be biased due to the usage of a finite length all-pass filter. This bias term can be decreased by increasing the length of the all-pass filter, $L_{a p}$.

\section{CONCLUSION}

In this paper we introduced a new approach for computing the minimum-phase filter and its associated all-pass filter in a computationally efficient manner using the fast QLfactorization. The proposed method convergences asymtocally toward the true filters with the complexity depending on the required precision.

\section{REFERENCES}

[1] J. G. Proakis and D. G Manolakis, Digital Signal Processing, Prentice Hall, 3 edition, 1995.

[2] W. H. Gerstacker, F. Obernosterer, Meyer R., and J. B. Huber, "On Prefilter Computation for Reduced-State Equalization," IEEE Trans. on Wireless Commun., vol. 1, pp. 793-800, Oct. 2002.

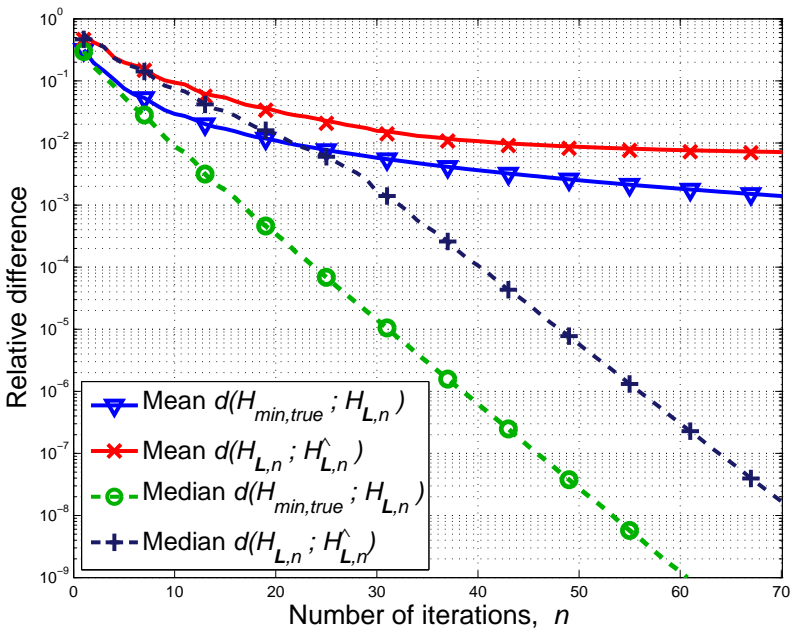

Fig. 4. HT0 profile, $L=10$. Mean and median value of the relative deviations, $d\left(\mathcal{H}_{\text {min,true }} ; \mathcal{H}_{L, n}\right)$ and $d\left(\mathcal{H}_{L, n} ; \widehat{\mathcal{H}}_{L, n}\right)$ when $L_{a p}=32$.

[3] J. F. Claerbout, Fundamentals of Geophysical Data Processing, Blackwell Scientific Publications, 1985.

[4] A. H. Sayed and T. Kailath, "A Survey of Spectral Factorization Methods," Numerical Linear Algebra with Applications, vol. 8, pp. 467-496, Jul. 2001.

[5] M. Hansen, L. P. B. Christensen, and O. Winther, "On Sphere Detection and Minimum-Phase Prefiltered Reduced-State Sequence Estimation," in GLOBECOM'07, 2007, pp. 4237-4241.

[6] M. Hansen, L. P. B. Christensen, and O. Winther, "Computing the Minimum-Phase Filter using the QLFactorization," Under preparation.

[7] N. Al-Dhahir and J. M. Cioffi, "MMSE DecisionFeedback Equalizers: Finite-Length Results," IEEE Trans. on Info. Theory, vol. 41, pp. 961-975, Jul. 1995.

[8] A. W. Bojanczyk, R. P. Brent, and F.R. de Hoog, "QR Factorization of Toeplitz Matrices," Numer. Math., vol. 49, pp. 81-94, Jul. 1986.

[9] D. R. Sweet, "Fast Toeplitz Orthogonalization," Numer. Math., vol. 43, pp. 1-21, Feb. 1984.

[10] S. Qiao, "Hybrid Algorithm for Fast Toeplitz Orthogonalization," Numer. Math., vol. 53, pp. 351-366, May 1988.

[11] D. R. Sweet, "Fast Block Toeplitz Orthogonalization," Numer. Math., vol. 58, pp. 613-629, 1991.

[12] 3GPP TSG GERAN 3GPP TS 45.005, Radio Transmission and Reception (Release 5). 